\title{
Night time versus daytime transient ischaemic attack and ischaemic stroke: a prospective study of 110 patients
}

\author{
Claudio Bassetti, Michael Aldrich
}

\begin{abstract}
Objective-Ischaemic stroke occurs only in $20 \%-40 \%$ of patients at night. The aim of the study was to compare sleep and stroke characteristics of patients with and without night time onset of acute ischaemic cerebrovascular events.

Methods-A consecutive series of 110 patients with transient ischaemic attack $(n=45)$ or acute ischaemic stroke $(n=65)$ was studied prospectively by means of a standard protocol which included assessment of time of onset of symptoms, sleep, and stroke characteristics. An overnight polysomnography was performed after the onset of transient ischaemic attack/ stroke in 71 patients. Stroke and sleep characteristics of patients with and without cerebrovascular events occuring at night (between midnight and 0600) were compared.
\end{abstract}

Results-A night time onset of transient ischaemic attack or stroke was reported by $23(21 \%)$ of 110 patients. Patients with daytime and night time events were similar in demographics; risk factors; associated vascular diseases; clinical and polysomnographic sleep characteristics (including severity of sleep apnoea); and stroke severity, aetiology, and outcome. Only the diastolic blood pressure at admission was significantly lower in patients with night time events $(74 v 82 \mathrm{~mm} \mathrm{Hg}$, $\mathrm{p}=\mathbf{0 . 0 1}$ ).

Conclusions-Patients with night time and daytime transient ischaemic attack/ stroke are similar in sleep and stroke characteristics. Diastolic hypotension may predispose to night time cerebrovascular events. Factors not assessed in this study probably account for the circadian variation in the frequency of transient ischaemic attack and acute ischaemic stroke.

(F Neurol Neurosurg Psychiatry 1999;67:463-467)

Keywords: stroke onset; sleep apnoea; circadian rhythm; blood pressure

Correspondence to: Dr Claudio Bassetti,

Department of Neurology, University

Hospital-Inselspital, 3010

Bern, Switzerland. Fax 0041

31632 9679; email

claudio.bassetti@insel.ch

Received 7 January 1999 and in revised form

5 May 1999

Accepted 13 May 1999
Possible explanations for the circadian pattern of cerebrovascular events have focused on circadian or postural changes in platelet aggregation, thrombolysis, blood pressure, heart rate, and catecholamine concentrations that occur after awakening with resumption of physical and mental activities.

In a minority of cases, which varies in the literature from less than $10 \%$ to as much as $44 \%$, stroke occurs at night. ${ }^{2-4}$ This suggests that sleep, although "protective" for most cerebrovascular events, may represent a vulnerable state for a subset of patients at risk for stroke. Nocturnal blood pressure swings, cardiac arrhythmias, and sleep disordered breathing have been suggested as possible explanations for the nocturnal onset of stroke.

In the absence of systematic studies on daytime and night time onset of cerebrovascular events we performed a prospective analysis of stroke and sleep characteristics in a consecutive series of 110 patients with transient ischaemic attack or acute ischaemic stroke.

\section{Patients and methods}

A consecutive series of 120 adult patients admitted to the University of Michigan Hospitals with acute (retinal or cerebral) transient ischaemic attack or ischaemic stroke-defined by standard criteria-were considered for the study. The study protocol was approved by the institutional review board of the University of Michigan. Subjects were examined with a standard protocol by one of us (CB) usually within the first 2 days after admission to hospital. When a reliable history could not be obtained from a subject because of aphasia or confusion, information was obtained from relatives. Reliable clinical information including the time of onset of transient ischaemic attack or stroke was obtained in $110(92 \%)$ of 120 patients. These 110 patients are the basis for the present analysis.

\section{STROKE ASSESSMENT}

Our stroke investigation included questions about such cardiovascular risk factors as family history of stroke, hypertension, diabetes, hypercholesterolaemia, smoking (pack-years), and alcohol consumption (drinks/week). Treatment with aspirin at the time of transient ischaemic attack/stroke onset was recorded. The presence of heart failure, coronary heart disease, and peripheral vascular disease was assessed. Estimated time of onset of transient ischaemic attack and stroke were noted. The time of onset for those patients who were well 
at bedtime but woke up with symptoms was the time of awakening. Night time events were defined as transient ischaemic attack and stroke occurring between midnight and 0600 . Clinical examination included assessment of body mass index and blood pressure at admission. Maximal severity of stroke was assessed with the Scandinavian stroke scale (range 0-58). ${ }^{5}$ The maximal score (no neurological deficit) is 58. A score $<30$ represents severe stroke. Stroke investigation included blood tests, 12 lead ECG, chest radiography, Doppler ultrasonography, and brain CT, or MRI, or both in all subjects. The presence of old strokes on brain CT or MRI was noted. Echocardiography was performed in $67(61 \%)$ and conventional or MR angiography in $47(43 \%)$ of 110 patients. Aetiology of stroke was classified according to the criteria of the Trial of Acute Stroke Treatment (TOAST) as large artery disease, small artery disease (microangiopathy), cardioembolism, other (carotid dissection), and unknown. ${ }^{6}$ Outcome was classified at discharge from the hospital as good (no deficits or deficits without restriction of lifestyle, independent), or poor (deficits with restriction of lifestyle, dependent).

SLEEP ASSESSMENT

We assessed sleep-wake habits and disturbances preceding transient ischaemic attack and stroke including estimated total sleep/24 hours, history of insomnia (disturbed or insufficient sleep), snoring, and hypersomnia (excessive daytime sleepiness). Snoring was considered habitual when it was reported to occur often or always. The habitual use of hypnotic drugs was also recorded. Sleepiness was estimated with the Epworth sleepiness scale. ${ }^{7}$ Polysomnography was recorded whenever possible and scored according to international criteria as described elsewhere. ${ }^{8}$ An overnight polysomnography was performed within a mean of 7 days (range 1-45 days) after the onset of the cerebrovascular event in $71(65 \%)$ of 110 patients. In the remaining 39 patients the examination was refused $(n=17)$ or unavailable $(n=22)$. The number of apnoeas and of apnoeas plus hypopnoeas/hour of sleep was expressed as apnoea index and apnoeahypopnoea index (AHI). We also noted maximal and average duration of respiratory events, minimal oxygen saturation $\left(\mathrm{SaO}_{2}\right)$ and percentage of sleep time spent with $\mathrm{SaO}_{2}<90 \%$.

STATISTICAL ANALYSIS

Nominal data were analysed with the $\chi^{2}$ test. For continuous data, we used the unpaired $t$ test and the Mann-Whitney $U$ test. Continuous values are given as mean (SD). Statistical significance was set at $\mathrm{p}<0.05$.

\section{Results}

There were 48 women and 62 men with a mean (SD) age of 59 (15) years (range 19-80). There were 45 patients with transient ischaemic attack and 65 with acute ischaemic stroke. The percentage of cerebrovascular events per 6 hour period were the following: $23(21 \%)$ between 0000 and 0559., $46(42 \%)$ between

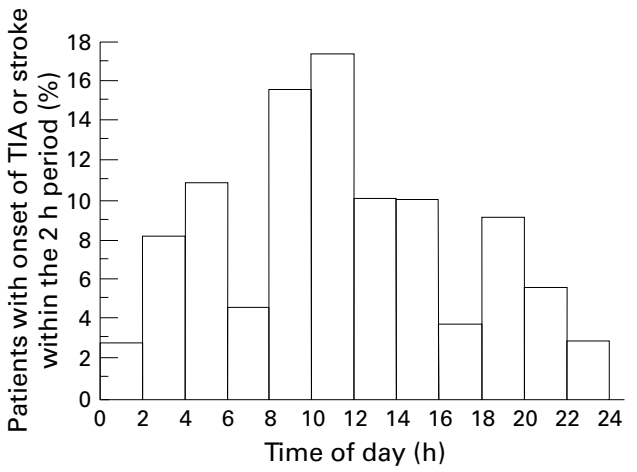

Time of onset of stroke and transient ischaemic attack in 110 consecutive patients.

Table 1 Stroke and transient ischaemic attack (TIA) at night and during the day: demographics and risk factors

\begin{tabular}{lll}
\hline & $\begin{array}{l}\text { Stroke/TIA at } \\
\text { night } n=23\end{array}$ & $\begin{array}{l}\text { Stroke/TIA } \\
\text { during the day } \\
n=87\end{array}$ \\
\hline Age & $63.1(13.4)$ & $58.0(15.5)$ \\
male:female & $15: 8$ & $47: 40$ \\
Body mass index & $28.4(5.8)$ & $28.4(7.6)$ \\
TIA:stroke & $8: 15$ & $37: 50$ \\
Hypertension & $14 / 23$ & $60 / 86$ \\
Blood pressure: & & \\
$\quad$ Systolic & $147.2(26.7)$ & $152.8(19.6)$ \\
$\quad$ Diastolic & $73.7(14.7)$ & $85.7(18.5) \ddagger$ \\
Diabetes & $9 / 23$ & $24 / 85$ \\
Smoking (pack-years) & 175 & 223 \\
Hypercholesterolaemia & $12 / 20$ & $42 / 84$ \\
Coronary heart disease & $6 / 22$ & $23 / 87$ \\
Heart failure & $6 / 21$ & $17 / 82$ \\
Peripheral vascular disease & $4 / 21$ & $19 / 85$ \\
Aspirin treatment $\dagger$ & $8 / 23$ & $31 / 86$ \\
\hline
\end{tabular}

Values in parentheses are SD. Only one $\ddagger$ of the differences was significant.

*At admission.

†At the time of TIA/stroke.

0600 and 1159, $26(24 \%)$ between 1200 and 1759 , and $14(13 \%)$ between 1800 and 2359 . This circadian pattern was similar in patients with transient ischaemic attack and stroke; eight $(18 \%)$ of the transient ischaemic attack patients and $15(23 \%)$ of the stroke patients had a night time onset of the cerebrovascular event). The distribution of time of onset of transient ischaemic attack/stroke in the 110 patients is shown in figure 1 .

CARDIOVASCULAR RISK FACTORS

Patients with $(n=23)$ and without $(n=87)$ night time onset of transient ischaemic attack/stroke were similar in all demographic variables, type of cerebrovascular event, risk factors, associated vascular diseases, and use of aspirin at the time of the cerebrovascular event (table 1). Only the diastolic blood pressure at admission was significantly lower in patients with night time onset of stroke ( $74 v 82 \mathrm{~mm} \mathrm{Hg}, \mathrm{p}=0.01)$.

\section{SLEEP CHARACTERISTICS}

All clinical sleep variables evaluated including history of habitual snoring (11 patients $(48 \%)$ v 43 patients $(49 \%)$ ) and hypersomnia (eight patients $(35 \%)$ v 33 patients $(38 \%))$; Epworth sleepiness score $(8.6(0.7)$ v $8.4(0.5))$; and habitual use of hypnotic drugs (three patients $(13 \%)$ v 11 patients (13\%)) preceding the acute cerebrovascular event were similar in patients with $(n=23)$ and without $(n=87)$ night 
Table 2 Stroke and TIA at night and during the day: Clinical sleep characteristics*

\begin{tabular}{lll}
\hline & $\begin{array}{l}\text { Stroke/TIA at } \\
\text { night } n=23\end{array}$ & $\begin{array}{l}\text { Stroke/TIA } \\
\text { during the day } \\
n=87\end{array}$ \\
\hline Estimated total sleep/24 h & $8.4(0.5) \mathrm{h}$ & $7.7(0.2) \mathrm{h}$ \\
Habitual snoringt & $11 / 23$ & $43 / 87$ \\
Hypersomnia† & $8 / 22$ & $32 / 84$ \\
Insomnia† & $10 / 22$ & $23 / 84$ \\
Epworth sleepiness score & $8.6(0.7)$ & $8.4(0.5)$ \\
Habitual use of hypnotics $\dagger$ & $3 / 20$ & $11 / 84$ \\
\hline
\end{tabular}

None of the differences between the two groups were statistically significant

$\star$ Preceding the onset of the acute cerebrovascular event, †oftenalways

Table 3 Stroke and transient ischaemic attack (TIA) at night and during the day: polysomnographic sleep characteristics*

\begin{tabular}{|c|c|c|}
\hline & $\begin{array}{l}\text { Stroke/TIA at } \\
\text { night } n=15\end{array}$ & $\begin{array}{l}\text { Stroke/TIA } \\
\text { during the day } \\
n=52\end{array}$ \\
\hline Interval to recording (days) & $10.6(3.4)$ & $6.7(0.9)$ \\
\hline Total sleep time (min) & $276.9(23.4)$ & $269.4(10.3)$ \\
\hline$\%$ NREM sleep 1 & $23.7(3.9)$ & $21.8(1.8)$ \\
\hline$\%$ NREM sleep 2 & $30.4(4.5)$ & $29.7(1.8)$ \\
\hline$\%$ NREM sleep 3-4 & $1.1(0.5)$ & $3.9(0.8)$ \\
\hline$\%$ REM sleep & $9.0(1.6)$ & $10.0(1.0)$ \\
\hline Apnoea-hypopnoea index & $22.9(9.9)$ & $27.4(4.2$ \\
\hline Apnoea index & $13.9(8.1)$ & $7.8(2.4)$ \\
\hline Minimal $\mathrm{SaO}_{2}{ }^{\star}$ & $85.5(1.4)$ & $80.8(1.5)$ \\
\hline Mean apnoea duration (s) & $17.4(1.2)$ & $17.1(1.0)$ \\
\hline
\end{tabular}

Values are mean (SD).

None of the differences between the two groups were statistically significant.

*After the onset of the acute cerebrovascular event.

$\mathrm{SaO}_{2}=$ oxygen saturation.

Table 4 Stroke at night and during the day: distribution, severity, and outcome

\begin{tabular}{lll}
\hline & $\begin{array}{l}\text { Stroke at night } \\
n=15\end{array}$ & $\begin{array}{l}\text { Stroke during the } \\
\text { day } n=50\end{array}$ \\
\hline Carotid:vertebrobasilar & $11: 2$ & $34: 13$ \\
Scandinavian stroke scale & $36.5 \pm 6.1$ & $40.2 \pm 2.2$ \\
Old strokes on CT & $3 / 11$ & $8 / 24$ \\
Good outcome & $7 / 13$ & $19 / 39$ \\
\hline
\end{tabular}

Values in parentheses are SD

None of the differences between the two groups were statistically significant.

*Independent at discharge from hospital.

time onset of transient ischaemic attack/stroke (table 2). Sleep disordered breathing and other polysomnographic sleep characteristics assessed after the acute cerebrovascular event were also similar in both groups (table 3 ). There was no significant difference in estimated onset time of transient ischaemic attack/ stroke when comparing patients with severe sleep apnoea (AHI>30, 12.5 (5.5)) and patients without sleep disordered breathing (11.0 (5.9)).

\section{STROKE CHARACTERISTICS}

Patients with $(n=15)$ and without $(n=50)$ night time onset of stroke were similar in most stroke characteristics evaluated including topography of stroke, Scandinavian stroke score (36.5 v 40.2 ), and outcome of stroke (table 4). The estimated time of onset of acute cerebrovascular events was also similar in the five different aetiology groups. Night time onset of transient ischaemic attack/stroke was somewhat more common in patients with microangiopathy than with other aetiologies of stroke (macroan-
Table 5 Estimated time of onset of stroke/transient ischaemic attack (TIA) according to aetiology

\begin{tabular}{ll}
\hline Aetiology & Stroke/TIA at night \\
\hline Macroangiopathy $(\mathrm{n}=22)$ & $5 / 22$ \\
Microangiopathy $(\mathrm{n}=14)$ & $4 / 14$ \\
Cardioembolic $(\mathrm{n}=14)$ & $3 / 14$ \\
Other causes $(\mathrm{n}=20)$ & $3 / 20$ \\
Unknown $(\mathrm{n}=36)$ & $7 / 36$
\end{tabular}

None of the differences between the groups were statistically significant.

${ }^{\star}$ Four patients with incomplete diagnostic work up and unkown aetiology of stroke were excluded from this analysis.

giopathy, cardioembolic, other, unknown (table 5). Old strokes were found on brain CT/MRI in about one fourth of patients with transient ischaemic attack and stroke in both patient groups (with and without nocturnal events).

\section{Discussion}

The present study assesses stroke and sleep characteristics in 110 consecutive patients with transient ischaemic attack or acute ischaemic stroke according to time of onset of the cerebrovascular event. As we studied a cohort admitted to hospital with an average age of 59 years our patients may not be fully representative of stroke and transient ischaemic attacks in the population. Despite this potential limitation, we confirm and expand previous data showing that not only acute ischaemic stroke but also transient ischaemic attack have a circadian pattern with a peak of onset between 0600 and 1200 and about $20 \%$ of events occurring at night time. ${ }^{9-11}$ Furthermore, it is shown for the first time that patients with daytime and night time onset of transient ischaemic attack/stroke are similar in most cardiovascular risk factors; clinical and polysomnographic sleep characteristics; and stroke parameters.

ONSET OF TRANSIENT ISCHAEMIC ATTACK/STROKE AND CARDIOVASCULAR RISK FACTORS

We found that patients with and without night time cerebrovascular events are similar in cardiovascular risk factors and associated vascular diseases. Similarly, Palomäki et al found that age, history of hypertension, diabetes, smoking, alcohol consumption, and body mass index did not differ in 59 male patients with sleep related and 108 male patients with sleep unrelated stroke. ${ }^{12}$ We also confirmed that aspirin does not modify the circadian pattern of stroke onset. ${ }^{9}$

The only difference found between patients with daytime and night time onset of transient ischaemic attack/stroke in our series was a lower diastolic blood pressure on admission in the second group. This finding suggests that low diastolic blood pressure values may predispose to night time onset of cerebrovascular events enhancing the sleep related fall in blood pressure. ${ }^{13}$ This finding is in line with previous reports suggesting a relation between night time hypotension and silent stroke ${ }^{14}$ and recurrent stroke. ${ }^{15}$

The link between blood pressure and stroke severity or outcome remains, conversely, unclear. Some studies have shown an adverse 
effect of high nocturnal blood pressure on outcome of cerebrovascular diseases. ${ }^{16}$ On the other hand, low admission blood pressure values found to correlate with lower stroke mortality and morbidity, ${ }^{17}{ }^{4}$ but also with worse outcome $^{18}$ and higher risk of stroke progression. ${ }^{19}$

ONSET OF TRANSIENT ISCHAEMIC

ATTACK/STROKE AND SLEEP CHARACTERISTICS.

Sleep disordered breathing is present in about $50 \%$ of patients with acute cerebrovascular diseases..$^{82021}$ Furthermore, at least one paper suggested habitual snoring as an independent risk factor for sleep related stroke. ${ }^{12}$ To our surprise, however, we did not find any significant difference in time of onset of cerebrovascular events in patients with and without sleep disordered breathing. This was also true when comparing patients with normal sleep breathing with patients with severe sleep apnoea (AHI>30). This finding confirms data presented in abstract form by Kapen et al in a series of 48 patients with acute stroke. ${ }^{22}$ Similarly, Spriggs et al found that a history of habitual snoring increases the risk of stroke but not the risk for strokes to occur at night. ${ }^{23}$ These data suggest that sleep apnoea-while being a risk factor for stroke $\mathrm{e}^{24-26}$ - may only rarely represent the immediate cause of transient ischaemic attack or stroke. Rather, respiratory events during sleep may cause haemodynamic and haematological changes which increase the risk of cerebrovascular events at the transition from sleep to wakefulness and during the subsequent few hours. This hypothesis is supported by the recent report of a decreased cerebrovascular reactivity in patients with obstructive sleep apnoea, which was more pronounced in the morning than in the afternoon. ${ }^{35}$ In the literature we found only one case report - presented in abstract form-of an acute cerebrovascular event occurring immediately after a witnessed apnoeic event. ${ }^{28}$ It is nevertheless conceivable that in a patient with severe sleep apnoea swings in blood pressure, decreased cerebral blood flow, and cardiac arrhythmias produced by respiratory events may trigger cardiovascular and cerebrovascular events during sleep. ${ }^{29-33}$

\section{ONSET OF TRANSIENT ISCHAEMIC}

ATTACK/STROKE AND STROKE CHARACTERISTICS In all characteristics assessed-including severity, topography, aetiology, and short term outcome of cerebrovascular events-patients with and without night time onset of transient ischaemic attack/stroke were found to be similar. It is noteworthy that in our study all stroke aetiologies had a morning peak of onset and that microangiopathy was the most common stroke subtype with a night time onset $(29 \%)$. In a study of 1233 patients Lago et al also found a higher frequency of all types of stroke during the day and a higher night time onset in macroangiopathy and microangiopathy compared with other stroke subtypes. ${ }^{34}$ Macroangiopathic and microangiopathic strokes may be particularly susceptible to nocturnal haemodynamic changes and decreased cerebral per- fusion (see above). Although yet to be proved, it is possible that intrathoracic pressure variations related to sleep apnoea may also predispose to cardioembolic strokes secondary to right-left shunt (for example, patent foramen ovale).

Whether or not stroke in sleep has a worse outcome remains unclear. We found a similar short term outcome in patients with night time and daytime stroke. However, patients with night time stroke, particularly when associated with sleep apnoea, were reported to have an increased 6 month and 4 year mortality and a worse 1 year functional outcome. ${ }^{202327}$

This research has some limitations. Firstly, and most importantly, the differentiation between events occurring at night time versus sleep and at daytime versus during wakefulness was not performed. For example, it is possible that some night time transient ischaemic attacks were noted only because they occurred during nocturnal awakenings. In addition, our data do not enable us to determine whether or not awakening from sleep may represent the single most crucial period for onset of cerebrovascular events. ${ }^{9}{ }^{12}$ Secondly, our study design does not exclude the possibility that the highest incidence in the early hours of the morning could be overestimated due to patients who awaken with stroke. Conversely, short lasting transient ischaemic attacks occurring in sleep may have been missed. Thirdly, differences in blood pressure values on admission need to be considered with caution considering the absence of data on time of blood pressure measurements. As blood pressure normally decreases at night it is conceivable that the lower blood pressure encountered in patients with night time onset events may merely reflect the normal circadian fall in blood pressure. Fourthly, because of the few patients, comparisons between different groups (for example, morning versus afternoon versus night time events) were not undertaken. For the same reason it is also possible that true differences between the two groups analysed may have been missed because of insufficient statistical power (type II error). Finally, it should be kept in mind that acute stroke may affect both sleep architecture and sleep breathing control (for further discussion see Bassetti and Aldrich ${ }^{21}$ ). Hence, the reported polysomnographic data may only in part reflect sleep characteristics preceding the acute cerebrovascular event.

In conclusion we have shown that (1) night time and daytime transient ischaemic attack/ stroke are similar in sleep and stroke characteristics, and (2) diastolic hypotension may predispose to night time cerebrovascular events. It is likely that the morning peak for onset of transient ischaemic attack and ischaemic stroke is related to several mechanisms. Factors not assessed in this study including sleep-wake related changes in fibrinolysis, platelet aggregatability, blood viscosity, catecholamine concentrations, and such haemodynamic variables as blood pressure and heart rate probably account for the circadian variation of cerebrovascular accidents and should be further investigated. 
We thank Dr Douglas Quint for reviewing the neuroradiological studies. This research was supported by the Swiss National Scistudies. This research was supported by
ence Foundation Grant 32-49853.96.

1 Elliott WJ. Circadian variation in the timing of stroke onset: a meta-analysis. Stroke 1998;29:992-6.

Marshall J. Diurnal variation in occurence of strokes. Stroke 1977;8:230-1.

3 van der Windt C, van Gijn J. Cerebral infarctions do not occur typically at night. $\mathcal{F}$ Neurol Neurosurg Psychiatry 1988; 51:109-11.

4 Chamorro A, Vila N, Ascaso C, et al. Blood pressure and functional recovery in acute ischemic stroke. Stroke 1998;29:1850-3.

5 Scandinavian Stroke Study Group. Multicenter trial of hemodilution in ischemic stroke: background and study protocol. Stroke 1985;16:885-90.

6 Adams HP, Bendixen BH, Kappelle LJ. Classification of subtype of acute ischemic stroke: definitions for use in a multicenter clinical trial. Stroke 1993;24:35-41.

7 Johns MW. Sleepiness in different situations measured by the Epworth sleepiness scale. Sleep 1994;17:703-10.

8 Bassetti C, Aldrich M, Chervin R, et al. Sleep apnea in the acute phase of TIA and stroke. Neurology 1996;47:116773 .

9 Marsh EE, Biller J, Adams HP, et al. Circadian variation in onset of acute ischemic stroke. Arch Neurol 1990;47:1178ons

10 Wroe SJ, Sandercock P, Bamford J, et al. Diurnal variation in incidence of stroke: Oxfordshire community stroke project. BMF 1992;304:155-7.

11 Haapaniemi H, Hillbom M, Juvela S. Weekend and holiday increase in the onset of ischemic stroke in young women. Stroke 1996;27:1023-7.

12 Palomäki H, Partinen $M$, Juvela $S$, et al. Snoring as a risk factor for sleep-related brain infarction. Stroke 1989;20: $1311-15$

13 Millar-Craig MW, Bishop CN, Raftery EB. Circadian variation of blood pressure. Lancet 1978;1:795-7.

14 Watanabe N, Imai Y, Nagai K, et al. Nocturnal blood pressure and silent cerebrovascular lesions in elderly Japanese. Stroke 1996;27:1319-27.

15 Nakamura K, Oita J, Yamaguchi T. Nocturnal blood pressure dip in stroke survivors. A pilot study. Stroke 1995. 26:1373-8.

16 Yamamoto Y, Akiguchi I, Oiwa K, et al. Adverse effect of nighttime blood pressure on the outcome of lacunar infarct nighttime blood pressure on the

17 Britton M, Carlsson A. Very high blood pressure in acute stroke. F Intern Med 1990;228:611-5.
18 Allen CMC. Predicting the outcome of acute stroke: a prognostic score. 7 Neurol Neurosurg Psychiatry 1984;47:475-80.

19 Jörgensen HS, Nakayama H, Raaschou HO, et al. Effect of blood pressure and diabetes on stroke in progression. Lancet 1994;344:156-9.

20 Dyken ME, Somers VK, Yamada T, et al. Investigating the elationship between stroke and obstructive sleep apnea. Stroke 1996;27:401-7.

21 Bassetti C, Aldrich MS. Sleep apnea. Final report on 128 patients. Sleep 1999;22:217-23.

22 Kapen S, Goldberg J, Diskin C, et al. The circadian rhythm of ischemic stroke and its relationship to obstructive sleep apnea. Sleep Research 1992;21:216.

23 Spriggs DA, French JM, Murdy JM, et al. Snoring increases the risk of stroke and adversely affects prognosis. $Q \mathcal{F} \mathrm{Med}$ 992;303:555-62.

24 Palomäki H, Partinen M, Erkinjuntti T, et al. Snoring, sleep apnea syndrome, and stroke. Neurology 1992;42:75-82.

25 Partinen M. Ischaemic stroke, snoring and ostructive sleep apnea. Fournal of Sleep Research 1995;4:156-159.

26 Bassetti C. Habitual snoring, sleep apnoea, and stroke prevention. F Neurol Neurosurg Psychiatry 1997;62:303.

27 Good DC, Henkle JQ, Gelber D, et al. Sleep-disordered breathing and poor functional outcome after stroke. Stroke 1996;27:252-9.

28 Rivest J, Reiher J. Transient ischemic attacks triggered by symptomatic sleep apneas [abstract]. Stroke 1987;18:293.

29 Balfours EM, Franklin KA. Impairment of cerebral perfusion during obstructive sleep apnea syndrome. $A m \mathcal{F}$ perfusion during obstructive sleep apn

30 McGinty D, Beahm E, Stern N, et al. Nocturnal hypotension in older men with sleep-related breathing disorders. Chest 1988;94:305-1

31 Netzer N, Werner P, Jochums I, et al. Blood flow of the middle cerebral artery with sleep-disordered breathing. Stroke 1998;29:87-93.

32 Pressman MR, Schetman WR, Figueroa WG, et al. Transient ischemic attacks and minor stroke during sleep. Stroke 1995;26:2361-5.

33 Jung HH, Bassetti C, Hess CW. Near cardiac death following REM-sleep: polysomnographic report in a patient with sleep apnea and narcolepsy. Fournal of Sleep Research 1997; $57-58$.

34 Lago A, Geffner D, Tembl J, et al. Circadian variation in acute ischemic stroke. A hospital-based study. Stroke 1998; 29:1873-5.

35 Diomedi, Placidi F, Cupini LM, et al. Cerebral hemodynamic changes in sleep apnea syndrome and effect of continuous positive airway pressure treatment. Neurology 1999;51:1051-6. 